Cinémas

Revue d'études cinématographiques

Journal of Film Studies

\title{
À nous la rue de Moustapha Dao : de la représentation à la présentification du réel
}

\section{Catherine Koassiwa Topou}

Volume 11, numéro 1, automne 2000

Écritures dans les cinémas d’Afrique noire

URI : https://id.erudit.org/iderudit/024832ar

DOI : https://doi.org/10.7202/024832ar

Aller au sommaire du numéro

Éditeur(s)

Cinémas

ISSN

1181-6945 (imprimé)

1705-6500 (numérique)

Découvrir la revue

Citer cet article

Koassiwa Topou, C. (2000). À nous la rue de Moustapha Dao : de la représentation à la présentification du réel. Cinémas, 11(1), 31-44. https://doi.org/10.7202/024832ar
Résumé de l'article

Parler de cinéma, est-ce nécessairement aborder la question de la représentation du réel? À tout le moins semble-t-il que les films des cinématographies africaines, films issus du réel, résistent à cette notion de représentation. Le film À nous la rue (1987), du cinéaste burkinabé Moustapha Dao, constituera le point de départ de notre investigation. Il permettra d'interroger, notamment, le concept de mimesis lié aux théories de l'esthétique occidentale. Notre propos est de démontrer que la compréhension d'un cinéma comme celui de Moustapha Dao, à l'instar des créations statuaires africaines, passe par le concept de présentification. 


\title{
À nous la rue de Moustapha Dao: de la représentation à la présentification du réel
}

\section{Catherine Koassiwa Topou}

\begin{abstract}
RÉSUME
Parler de cinéma, est-ce nécessairement aborder la question de la représentation du réel? A tout le moins semble-t-il que les films des cinématographies africaines, films issus du réel, résistent à cette notion de représentation. Le film $A$ nous la rue (1987), du cinéaste burkinabé Moustapha Dao, constituera le point de départ de notre investigation. Il permettra d'interroger, notamment, le concept de mimesis lié aux théories de l'esthétique occidentale. Notre propos est de démontrer que la compréhension d'un cinéma comme celui de Moustapha Dao, à l'instar des créations statuaires africaines, passe par le concept de présentification.
\end{abstract}

\footnotetext{
ABSTRACT

Does any discussion about cinema necessarily imply a discussion of the question of the representation of reality? It seems, at the very least, that films from various African cinemas, although stemming from reality, resist this notion of representation. $\dot{A}$ nous la rue (1987), directed by Burkina Faso filmmaker Moustapha Dao, will serve as the starting point of our research. Ir will allow us to question, among other issues, the concept of mimesis as developed by Western aesthetic theories. Our intention is to show that the understanding of a cinema such as Moustapha Dao's (as of African statuary creations) involves the concept of presentification.
} 
Dans la civilisation subsaharienne, le réel du monde sensible n'apparaît pas comme le fondement des créations religieuses. Ces créations culturelles, désignées communément par l'expression "arts africains traditionnels", n'imitent pas les apparences du réel; elles transfigurent le réel, elles donnent à voir un réel non apparent, invisible. Dans Art africain et esthétique occidentale, Roger Somé parle à propos des créations lobi et dagara du Burkina Faso de présentification. Il emprunte ce terme à JeanPierre Vernant qui l'utilise pour l'art grec dans un article intitulé «De la présentification de l'invisible à l'imitation des apparences». Pour Somé, les statues lobi et dagara ne représentent pas un sujet ou un objet ayant déjà une forme propre reconnaissable de tous, mais donnent une figure à un réel autre, celui de l'invisible, qui n'est vu ni connu de personne. La présentification devient alors la figuration de l'invisible. Elle rend compte de ce que la représentation ne peut faire, elle qui est du domaine des apparences, du visible. Qu'en est-il maintenant du cinéma africain ? Peut-on le comprendre à la lumière de ce concept de présentification? Il s'agit ici de voir si le concept de présentification s'applique à un cinéma comme celui du cinéaste burkinabé Moustapha Dao, et ce, à partir de son film $A$ nous la rue (1987).

\section{Une esthétique de l'hybride}

Moustapha Dao, dans son court métrage, a capté le quotidien d'enfants à la sortie de l'école. Un écolier sonne le gong: il est l'heure pour les enfants de quitter l'école et de vaquer à leurs occupations quotidiennes. Certains jouent au balafon pendant que les filles dansent. Deux des garçons se font gronder par leur père et apportent des balafons au marchand. Modibo et ses amis décident de construire des jouets en fil de fer et en bois. Les filles font des confiseries pour les vendre dans la rue. D'autres garçons jouent au football mais se font confisquer le ballon par une dame, qui le leur rend grâce aux larmes d'un enfant. La sœur de Modibo qui dansait est appelée par son père pour réviser ses leçons. Oussou fait le mur pour rejoindre ses copains et se bat avec Modibo pour l'amour de Sally, la vendeuse de bonbons. Les mères des deux garçons se disputent sous l'œil des enfants qui cessent de se battre et 
courent rejoindre les autres en entendant la musique. Garçons et filles se mettent à danser ensemble.

$A$ nous la rue n'est pas, en soi, une fiction au sens traditionnel de ce terme. En effet, la construction du scénario ne relève pas totalement de l'intrigue. L'objectif du cinéaste n'est pas seulement de nous conter une histoire sur les enfants de Ouagadougou, mais aussi et surtout de nous montrer leur quotidien. Le film consiste donc en une succession de petites scènes ou de microévénements mettant en scène des enfants s'appropriant la rue. Il ne raconte pas d'histoire dans la mesure où aucune véritable "dramatisation" ne le construit; il montre la réalité quotidienne de ces enfants.

Cette première analyse, qui révèle le parti pris esthétique de Moustapha Dao, pose la question de la représentation du réel. Dao expose ce qui n'est pas vu habituellement en travaillant avec la monstration :

Une sorte de plus-value sémantique est attribuée aux éléments ainsi montrés: au signifié dénoté par l'image s'ajoute, sous forme d'un coefficient de majoration et selon le principe d'une modalisation "intensificatrice", le signifié qu'on pourrait formuler ainsi: «ceci est important $\%$. On notera que cet effet de sens a partie liée avec le mode d'énonciation: c'est essentiellement la suspension du flux narratif et le débrayage qui l'accompagne qui donnent à l'acte de monstration un caractère ostentatoire (Gardies, 1993, p. 82).

Dans $A$ nous la rue, Moustapha Dao associe des plans appartenant au réel et des images de fiction. Il assemble des formes de façon que la technique cinématographique donne à voir des figures théoriquement antagoniques: la fiction et le documentaire. Une esthétique de l'hybride ne se satisfait pas d'une telle dichotomie. Ainsi l'espace filmique crée par Dao: nous ne sommes plus tout à fait dans une fiction, pas davantage dans un documentaire. Mais où sommes-nous?

La question s'est posée dès l'origine du cinéma africain. Dans son premier film, Borom Sarret (1963), Ousmane Sembène combine aussi images documentarisantes et fictionnelles: les plans du charretier donnent lieu à un tour d'horizon de Dakar 
et de sa population. Il en va de même pour le premier film africain, Afrique sur Seine (1957), fiction dans laquelle Paulin Vieyra et Mamadou Sarr incluent des vues de Paris. Le cinéma africain "réaliste" a eu comme objectif de reconquérir une identité perdue. Dès le début, cette cinématographie a emprunté une voie fondamentalement réaliste. Le réel cinématographique permettait de rétablir la vérité sur les peuples d'Afrique, les récits exotiques et ethnologiques ne privilégiant que des aspects fort limités de leur réalité. Plus de trente ans après la fin de la colonisation, le nouveau "combat" de cinéastes comme Moustapha Dao consiste à faire face aux images de guerre et de famine véhiculées par les médias occidentaux.

Dans $A$ nous la rue, Moustapha Dao s'attache à montrer des images et des gestes qui font partie de la vie quotidienne des enfants. Il y a là une véritable écriture de l'image dans le quotidien. Il observe les attitudes de ces enfants et les restitue grâce à la technique du cinéma. Lécriture de Dao met en jeu deux dispositions de filmage: plans volés au réel; insertion de plans de gestes artisanaux.

Les images "documentaires" sont aisément repérables, nombre d'indices les portant à notre attention. Ainsi, les regards à la caméra indiquent que les enfants ne sont pas à proprement parler des personnages de film, mais qu'ils vaquent à leurs activités quotidiennes. On note également des obstructions du champ de la caméra: dans plusieurs plans, un jeune garçon court et masque, l'espace d'un moment, la vision du champ. L'absence de continuité temporelle dans la scène de l'école constitue un troisième indice: parmi les derniers plans de la séquence, certains présentent une coloration à dominante jaune parce que, manifestement, ils ont été tournés alors que le soleil se couchait. En soi, ces indices tuent la représentation. Ils cassent la transparence telle qu'elle est conçue dans le cinéma dominant, même documentaire.

Les plans de gestes artisanaux quant à eux sont emblématiques de l'esthétique de l'hybride, car à l'intérieur d'un seul plan sont associées images documentaires et images fictionnelles, et ce, dans la volonté de montrer le réel. Très souvent, les inserts sont des gros plans portant sur des détails de fabrication de 
divers objets: un balafon, des jouets en fil de fer et en bois, des confiseries, etc. Ainsi, un bonbon donne lieu à une saynète retraçant les étapes de sa fabrication. Tout d'abord, la préparation est mise sur le feu pour faire fondre le caramel, ensuite la pâte est posée dans une assiette et, enfin, le caramel est roulé en boulettes. Les objets dont la fabrication vient d'être terminée sont mis en valeur par des plans à part qui, littéralement, les exposent, car en aucun moment ces plans ne sont dramatisés. En soi, cette absence de dramatisation dans des images qui appartiennent pourtant à la fiction - elles ont été construites pour le film — pousse à nous interroger sur le mode de lecture adapté à ce film.

Les plans de tambours, à la fin du film, sont produits par la même procédure de filmage. Si le résultat n'est pas le même, c'est que la musique les intègre immédiatement à la fiction. Néanmoins, ce sont dans ces inserts de plans de gestes artisanaux que se trouvent indissolublement liées la fiction et la réalité. L'écriture de ces plans impose donc au spectateur de réfléchir sur les possibilités de l'esthétique de l'hybride, celles d'une certaine ambivalence entre cinéma fictionnel et cinéma documentaire.

Moustapha Dao, dans son second film, Le Neveu du peintre (1988), raconte l'histoire du jeune Ali partant en vacances chez son oncle dans la grande ville de Ouagadougou. Il utilise le même filmage que pour $A$ nous la rue: au groupe de garçons fabriquant des jouets en bois et en fil de fer se substitue un groupe d'enfants construisant de faux pistolets. Le cinéaste filme la fabrication du revolver comme celle des jouets, étape par étape: le redressement de la tige à coups de marteau, enclenchement de l'arme au moyen d'une allumette, etc.

Cette esthétique de l'hybride n'est pas propre à Moustapha Dao. Elle se retrouve dans une grande majorité de films africains. Dans son court métrage Yiri Kan (1989), le cinéaste burkinabé Issiaka Konaté retrace l'initiation d'un garçon à la facture du balafon. Il ne s'agit pas véritablement d'une histoire, mais plutôt d'un trajet au cours duquel se manifestent certains microévénements. Le cinéaste filme des gestes de la vie quotidienne qui, traditionnellement, jalonnent le parcours initiatique. Issiaka Konaté utilise le même procédé que Moustapha Dao: il insère 
des plans de gestes artisanaux en retraçant les différentes étapes de construction de cet instrument de musique.

Comment cette hybridation cinématographique, cette rencontre du cinéma documentaire et du cinéma de fiction remetelle en cause le concept de représentation?

\section{De la représentation du réel à sa présentification}

Dès le prégénérique, $A$ nous la rue se démarque du cinéma de fiction traditionnel, donc de l'idée de représentation du réel liée à une écriture de la transparence. La fin du prégénérique montre les enfants en train de regarder un film burlesque en noir et blanc à la télévision. Au moment où sur l'écran le mot fin apparaît commence le générique du film de Dao. Ici, le cinéaste différencie déjà son cinéma de celui montré à la télévision.

Dans $A$ nous la rue, nous ne sommes pas au spectacle, mais dans la vie de tous les jours. Les enfants regardent un film qui, lui, est du cinéma, et pas n'importe lequel: du burlesque! En se distanciant de cette fiction télévisuelle, Dao ancre son film dans une critique entre le réel représenté et le réel qui s'offrirait à voir. Mais cette séance de projection est à la fois une mise en abîme du rôle du spectateur intrinsèque (les enfants) et extrinsèque au film (nous). En effet, les deux instances de réception s'entrecroisent dans leur mode de lecture. Spectateurs extrinsèques à la diégèse filmique, nous sommes aussi dans la même situation que les enfants du film: nous voyons une fiction. Pourtant, contrairement à eux, nous n'assistons pas à une représentation de la réalité comme c'est le cas dans le film burlesque, mais à la réalité même mise en images par la médiation de la technique du cinéma. La technique cinématographique permet ici de "reproduire» le réel, de le donner à voir. Dans Souko, un cinématographe en carton (1997), des enfants fabriquent une camera obscura en carton. L'un d'eux dessine un cheval et projette l'image sur un écran. Le cheval prend vie et traverse la toile. La confrontation avec l'imaginaire pur, qui relève de la fiction, permet de mieux observer ce qui dépend du réel. La réalité de la vie de ces enfants est d'autant plus grande qu'elle est confrontée avec l'irréalité vraie: l'imaginaire.

Il nous parait particulièrement important dans cette étude de distinguer la reproduction du réel et la représentation du réel. 
La représentation de la réalité est le fruit de l'activité créatrice du cinéaste, qui pour les besoins de son œuvre idéalise, sublime, modifie et crée un réel. Le cinéma a au moins une double potentialité de départ: la reproduction, captation directe du réel, et la représentation, création d'un réel imaginé ou imaginaire. De ces deux possibilités découleront aussi des conceptions différentes du média. Le cinéma de Moustapha Dao procède davantage du principe de reproduction, celui qui entraîne la monstration et par conséquent la présentification du réel. Dans une perspective ontologique, il faut distinguer le cinéma de Moustapha Dao du cinéma de fiction dominant, ce dernier représentant un réel à partir des principes de l'illusion. Par exemple, dans une fiction traditionnelle, il y aura connivence entre le destinataire et le réalisateur, le pacte de lecture faisant en sorte que le premier considère ce qu'il voit à l'écran comme irréel en même temps qu'il le prend pour réel. Nous sommes alors entièrement du côté de la représentation. La fiction traditionnelle n'a pas d'autre intérêt que de représenter une histoire qui n'a pas pour fonction d'être la réalité, mais son illusion. Mais l'image fictionnelle représente-t-elle toujours la réalité, est-elle toujours de l'ordre de l'illusion?

Moustapha Dao nous montre qu'une image fictionnelle, en droit, ne participe pas de la représentation. Le cinéaste ne manie plus les images comme un moyen de représentation de la réalité. Les images de fiction font apparaître des "signes» de la réalité africaine, de la même manière que celles issues directement du réel. Dans $A$ nous la rue, le réel ne réside plus simplement dans les plans volés ou dans les images de gestes artisanaux. Il est partout, dans tous les plans. Chaque personne, chaque lieu, chaque action nous montre une réalité.

Dao n'est pas un cinéaste qui se permet une certaine liberté par rapport au réel; son objectif est de montrer la quotidienneté. Le quotidien de la réalité africaine devient ainsi présent dans chaque plan du film. Et tous les niveaux de réception ne parlent plus d'identification, mais de participation à cette vie connue et reconnue. Les enfants qui marchent dans la rue nous montrent certaines routes de Ouagadougou. La mère qui fait la cuisine dans la cour expose la réalité de la cuisine traditionnelle 
africaine... La prise de vue cinématographique se confond avec une prise de la vie réelle, gommant ainsi toute évocation du point de vue du cinéaste. Le cinéma renvoie à un réel existant que l'appareil cinématographique ne fait que restituer dans la fidélité de ses parties non visibles. Les images de construits fictionnels sont dotées, tout comme les images documentarisantes, "d'indices» du réel. Ainsi le concept de représentation devient-il inadéquat même pour les images fictionnelles, car ces images, comme les images du réel, ne font plus appel à l'illusion mais à l'essence.

\section{Une écriture vouée à la présentification}

La tradition artistique occidentale s'ancre dans la recherche de la mimesis la plus parfaite, cela est aujourd'hui indiscutable. La création de cette machine parfaite qu'est le cinématographe a fortement servi cette recherche d'enregistrement de faits issus de la réalité mouvante. L'évolution des premières techniques, placée sous le sceau de la reproduction de la perspective et du mouvement, a beaucoup à voir avec ce rôle dévolu au cinématographe : la recherche du réel. Plusieurs formes d'expression artistique (peinture, thêâtre, etc.) étaient déjà engagées dans cette voie. Il n'y a donc pas de quoi s'étonner si le cinéma figure en bonne place dans l'histoire de la représentation dans les arts. Cela va de soi puisqu'il en découle. C'est très rapidement que la fiction, de simple possibilité qu'elle était dans les films des premiers temps, a été élevée au rang de norme.

$\grave{A}$ nous la rue relève d'une conceptualisation subvertissant la mimesis. Malgré le fait qu'il dévoile le réel, il ne se présente pas comme son imitation, comme une représentation de celui-ci. L'absence de mimesis dans $A$ nous la rue se manifeste dans la mise en images du film. Si on considère l'intentionnalité de Dao comme sujet, on constate qu'il ne raconte pas seulement une histoire qui serait la mise en images d'une fiction inventée ou la construction du réel autorisant le mensonge, la représentation. En soi, son choix de mêler les images documentarisantes à des images fictionnalisantes détruit le principe de représentation, car l'absence de fluidité de l'écriture empêche le destinataire de se laisser prendre aux illusions des images. La composition du film 
maintient le spectateur en état d'alerte, condition essentielle à la critique de la société présentée.

Dans $A$ ̀ nous la rue, Dao se munit d'images marquées «d'indices indiscutables du réel». Ces indices, empreintes de l'authenticité des images par rapport au réel, créent un paradoxe. Ces indices du réel sont des signes d'énonciation qui légitiment un sujet, quelqu'un filmant ces images. Mais même si l'énoncé est capable de s'effacer

\section{[...] devant l'instance énonciatrice, dont l'intention communicative prend le dessus sur "l'histoire", détrui- sant [ainsi] l'illusion de réel et l'identification du spec- tateur avec ce qui se passe sur l'écran, [et cela] con- trairement au cinéma traditionnel qui tend à masquer l'aspect "communicationnel " pour se manifester comme " histoire" pure [...] (Coremans, p. 78),}

ici aussi, les images qui sont données à voir détiennent une force, celle de leur symbole, identique à la vie.

Même si Dao en avait eu le désir, $A$ nous la rue ne pourrait être perçu comme une fiction pure, car la réalité contenue dans les images subvertit l'écriture fictionnelle. Le film est et se revendique comme un film de monstration, un "faire venir" et non de narration. À la suite d'André Gaudreault (1988, p. 91), nous entendons par monstration le fait de donner à voir le réel, de montrer des actions plutôt que de les raconter. La monstration "[caractérise] et [identifie] ce mode de communication d'une histoire qui consiste à montrer des personnages qui agissent plutôt qu’à dire ces péripéties qu'ils subissent \%.

Le cinéma axé sur une esthétique de l'hybride, tel que le conçoit Moustapha Dao, ne devra donc rien à la mimesis puisque, en ce qui concerne le réalisateur, la question initiale n'est plus de ressembler au monde qui existe l'espace d'un moment cinématographique, mais de donner à voir une réalité africaine telle qu'elle est et se trouve en dehors des espaces de spectacle. La réalité est captée; elle n'est pas le jeu de l'illusion et de l'apparence. Le concept de mimesis ne peut donc, dans le cas présent, s'accorder avec le film de Moustapha Dao. À aucun moment, le film ne veut être dans le ressemblant, dans le mime cinématographique de la vie. Au contraire, il est «le même» avec la vie qu’il 
donne à voir. À ce stade-ci, on peut donc soutenir que le concept de représentation devient artificiel lorsque l'on s'attaque à un cinéma fortement ancré dans le réel. Si ce terme de représentation s'applique au cinéma dominant, l'esthétique de l'hybride le remet en question.

On l'a vu, les principes de la monstration permettent à Moustapha Dao d'utiliser la technique cinématographique en vue d'une médiation. La monstration n'est pas une simple description de la vie, celle des enfants dans le réel quotidien. Comme principe, la monstration donne à voir ce que l'œil nu ne saurait voir. Elle devient l'espace fort d'un faire venir présentifiant l'invisible, la réalité non accessible à travers le simple langage. Cette monstration de la réalité transcende la réalité ellemême. Elle débouche sur quelque chose qui surpasse le visible. En donnant à voir la vie quotidienne d'enfants de Ouagadougou, Moustapha Dao ne conduit-il pas à une réflexion à laquelle ne saurait prétendre la simple description?

On a vu également que la monstration ne relève pas exclusivement de l'illusion. En donnant à voir le réel dans cette forme de cinéma hybride, Dao va plus loin que la simple représentation du réel. Il dépasse les qualifications formelles: documentaire ou fiction. La présentification instaure un espace de rencontre entre les formes, un lieu où fiction et documentaire se trouvent intimement liés. Ainsi, parler uniquement de l'aspect documentaire serait omettre la particularité même du film, qui repose sur la rencontre, la médiation entre les formes, les genres. C'est ce mélange, en lui-même, qui relève de la présentification.

Le quotidien des enfants est capté par la médiation de la technique cinématographique. Dans ce film, Dao donne une figure, une image au réel, et cette image procède du symbole. L'image montrée, qu'elle appartienne à un construit documentarisant ou fictionnalisant, devient un symbole du réel. Et c'est par cette dimension symbolique que l'essence se manifeste. Moustapha Dao, cinéaste, se rapproche ainsi du créateur de masques ou de statues car, comme lui, il cherche à travers ses créations à atteindre l'essence. «L'objet d'art que l'artiste parvient à produire est lui-même un signe, un symbole. Ce qu'il cherche à atteindre, c'est non pas l'apparence externe mais une essence" (Sylla, 
1988, p. 244). Par la monstration, Dao capte l'essence d'un peuple, la réalité de la vie. Grâce au cinéma, l'essence de cette vie apparaît, s'expose. Les capacités de la technique deviennent révélatrices de l'essence. Dao, en privilégiant les images appartenant au quotidien, arrive à les sublimer. Par la monstration, il atteint l'essence d'un peuple, un non-visible, et non simplement sa simple apparence extérieure. En cela, $A$ nous la rue relève non pas d'une représentation de la réalité mais de la présentification de celle-ci, car la présentification se définit, comme nous l'avons vu pour les créations africaines, par le fait qu'est donnée à voir une image symbolique incarnant la réalité. La présentification révèle les choses telles qu'elles sont. Elle est de l'ordre de l'essence, contrairement à la représentation qui est de l'ordre des apparences, de l'imaginaire et de l'imagination.

\section{De l'intérieur à l'extérieur, de l'invisible au visible}

Le cinéma capte la réalité pour l'exposer dans un dehors qui est celui de l'image. En soi, le cinéma serait donc, aussi, une ouverture, un extérieur. Il y aura transposition de la réalité première, intérieure, vers une réalité seconde, extérieure et cinématographique. Ainsi, le cinéma déplacerait le réel vers d'autres dimensions lorsqu'il est capté et projeté. Et comme le souligna Jean-Paul Sartre (1940, p. 128), "l'image est symbolique par essence et dans sa structure même, [si bien] qu'on ne saurait jamais supprimer la fonction symbolique d'une image sans faire s'évanouir l'image elle-même".

Par la médiation cinématographique, la réalité captée et projetée devient image. Cette image n'existe pas dans le monde réel. Elle ne prend nom et place qu'au cinéma, dans cette situation précise. Le réel cinématographique est imagé par l'objectif de la caméra et par la projection, des liens tout techniques. L'image cinématographique est alors ce symbole de la vie. Bien entendu, nous entendons par symbole un autre pareil. L'image cinématographique de la vie est la vie, mais au cinéma.

Dans À nous la rue, Dao présentifie le réel, car il va de la réalité intérieure d'un peuple vers l'extérieur, celui de l'image. La réalité intérieure d'un peuple, c'est tout ce qui se rattache aux personnes, aussi bien leur quotidien ou leurs habitudes que ce 
qu'elles font dans l'intimité de leur foyer ou encore dans la rue qui, bien qu'elle soit publique, est l'objet de confrontations privées: le père qui gronde ses enfants pour qu'ils aillent vendre le balafon, les deux mères qui se disputent à cause de leurs fils. Ce qui appartient normalement à l'intérieur de la famille est rendu public.

Moustapha Dao, au même titre que le créateur de masques ou de statues, peut être considéré comme un passeur. Il permet le passage. Quand la réalité est transcrite au cinéma, il y a passage entre le réel et le symbolique, passage entre la vie et l'image de la vie. Moustapha Dao est une sorte de médiateur entre le réel et sa présentification. Il est celui qui lie les deux et en fait une figure propre. Ainsi, à l'instar des statues, les images de son film permettent de rendre visible l'invisible. Nous entendons donc par invisible non ce qui appartient à une autre réalité, mais ce qui appartient à la même réalité, au réel apparent. Seulement, ce réel n'est vu ni connu de personne. Autrement dit, il reste à révéler.

Grâce à la technique du cinématographe, elle-même en adéquation avec les modes d'écriture traditionnels africains, le cinéma permet de révéler ce qui est caché, non vu. Le quotidien de ces enfants, leurs activités, leurs loisirs ne sont connus que d'eux seuls. Que font les enfants après l'école, au moment où ils sont libres de toute contrainte scolaire? Travaillant avec la monstration, Moustapha Dao donne à voir ce qui n'est pas vu habituellement.

La monstration du processus de fabrication des objets relève aussi du passage de l'invisible au visible, du caché au vu. En effet, les étapes de fabrication des objets appartiennent normalement aux coulisses, à ce qui ne se voit pas. A nous la rue montre néanmoins comment sont fabriqués des produits qui, généralement, ne sont vus qu'au moment où ils seront des produits finis. Chaque geste des enfants est un geste de dévoilement de la fabrication. Par ce processus de création, de fabrication des objets, Moustapha Dao interroge aussi le processus de création cinématographique. Toute œuvre parle toujours un peu d'elle-même. En nous montrant ces images de fabrication, il dévoile et rend visible le processus de fabrication de son film. Ici encore, ce pro- 
cessus vient remettre en cause l'idée d'une représentation basée sur une écriture cinématographique de la transparence.

Le concept de présentification semble être la pierre angulaire du cinéma d'Afrique noire. Il faut montrer la vie, préoccupation majeure de cette cinématographie. Mais il faut montrer d'elle les côtés souvent occultés par les films qui montraient l'Afrique. $\mathrm{La}$ présentification cinématographique donne ainsi des images d'un quotidien méconnu. Et Moustapha Dao, dans ses "fictions", donne à voir ce qui normalement, du fait de sa banalité, n'intéresse personne: le quotidien. Il montre ce qui normalement n'est pas donné à voir au cinéma, et surtout pas dans les films de fiction traditionnels, insistant chaque fois sur le processus de dévoilement de quelques autres choses cachées, et ce, même dans la diégèse. Par exemple, Modibo et ses amis chantent une chanson, s'accompagnant d'instruments de leur fabrication. Puis, dès que la bande de Modibo se déplace, ce qui était caché devient visible. Des jeunes filles qui dansent, jusque-là cachées, deviennent visibles au fur et à mesure que les garçons se déplacent. La troupe de chanteurs part et laisse la place aux jeunes danseuses. Ce plan fait apparaître de manière certaine que le filmage se voulait dévoilement diégétique. C'est de la même manière qu’à plusieurs reprises le film nous montre l'intimité des personnes, ce qui normalement est caché par les murs qui séparent le privé du public, le non vu de l'exposé. La caméra, comme Oussou, "fait le mur" pour offrir à notre regard ce qui se trouve derrière la rue: l'intérieur des foyers.

On peut donc soutenir que le concept de représentation du réel au cinéma appartient au discours du cinéma dominant. Il permet l'illusion et par là le mensonge. Le film de Moustapha Dao nous a permis de démontrer que ce concept ne convient pas à toute cinématographie. Et si tel est le cas, c'est que l'hybridité esthétique à l'œuvre dans ce cinéma répondrait, non "à des principes différents de ceux qui président aux impératifs de l'illusion-représentation" (Gardies et Haffner, 1987, p. 171), mais à ceux d'une présentification voulant révéler l'autre côté du réel.

Dans la présente étude, le cinéma de Moustapha Dao a été abordé comme un média qui, à l'instar des créations africaines 
traditionnelles, se fait l'intermédiaire entre l'intérieur et l'extérieur, le visible et l'invisible. Et nous avons considéré $A$ nous la rue en relation avec le concept de présentification, comme une forme artistique propre à une certaine forme de cinéma qualifié d'hybride. Mais nous avons passé sous silence ce que cette écriture cinématographique peut impliquer de poésie. En effet, l'hybridation cinématographique de $A$ nous la rue se hisse, par ses images appartenant au quotidien, au niveau de la poésie. Dans Ciné-journal, Jonas Mekas (1992, p. 259) parle « [d’un] réalisme [...] hissé au niveau d'intensité poétique où les significations deviennent plus profondes, universelles et à plusieurs niveaux ". Ces propos peuvent s'appliquer au film de Moustapha Dao. Ils ouvrent la voie à une étude plus vaste qui en ferait ressortir la force poétique. Mais définitivement, il faudra penser à affiner les concepts par l'intermédiaire desquels nous recevons et comprenons les films, à tout le moins certains films.

\section{Université de Paris III (Sorbonne Nouvelle)}

\section{OUVRAGES CITÉS}

Coremans, Linda. La Transformation filmique. Du contesto à Cadaveri Eccelenti. New York: Peter Lang, 1990.

Gardies, André. L'Espace au cinéma. Paris: Méridiens Klincksieck, 1993.

Gardies, André et Pierre Haffner (direction). Regard sur le cinéma négro-africain. Bruxelles: OCIC, 1987.

Gaudreault, André. Du littéraire au filmique. Système du récit. Paris: Méridiens Klincksieck, 1988.

Mekas, Jonas. Ciné-journal. Un nouveau cinéma américain (1959-1971). Paris: Éditions Paris expérimental, 1992.

Sartre, Jean-Paul. L'Imaginaire. Psychologie phénoménologique de l'imagination. Paris: Gallimard, 1940.

Somé, Roger. Art africain et esthétique occidentale. La statuaire lobi et dagara au Burkina Faso. Paris : L'Harmattan, 1998.

Sylla, Abdou. Créations et imitation dans l'art africain. Dakar: Université Cheikh Anta Diop, 1988.

Vernant, Jean-Pierre. “De la présentification de l'invisible à l'imitation de l'apparence ", Image et signification. Paris : La Documentation française, 1983. 